\title{
Estereotipos de género: Perspectivas en profesiones artesanales de Portugal
}

\author{
Fanny Monserrate Tubay ${ }^{1,2}$ (iD) 0000-0002-9156-0956 \\ 'Universidad Nacional de Educación a Distancia, Madrid, España. \\ infouned@adm.uned.es \\ Universidad de Lisboa, CIEG Centro Interdisicplinar de Estudios de Género, Lisboa,
} Portugal.

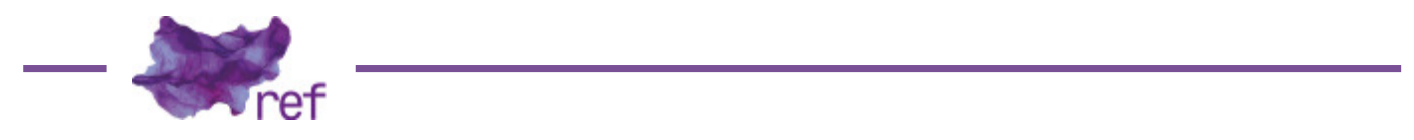

Resumen: Este artículo explora los estereotipos de género en una asociación de artesanos en Lisboa, a través de un estudio exploratorio de corte etnográfico, cuyos aportes dan cuenta del origen y las transformaciones de la profesión, así como de un conjunto de articulaciones que categorizan lo femenino y masculino. Los resultados exponen la dinámica de naturalización de los roles y la interseccionalidad de sus implicaciones en el contexto sociocultural. El trabajo muestra, por un lado, como se reafirman las desigualdades por el hecho de ser mujer u hombre, y revela, por otro, el trabajo que realizan las personas vinculadas, con el afán de deconstruir esos mecanismos y las ideas preconcebidas que los bordean.

Palabras claves: artesanos; estereotipos de género; interseccionalidad; mujeres; hombres.

\section{Gender stereotypes: Perspectives in the craft professions in Portugal}

Abstract: This article explores gender stereotypes in an artisans' association in Lisbon, through an exploratory study of ethnographic character, the contributions of which explain the origin and transformations of the profession, as well as a set of articulations that categorize the feminine and the masculine. The results reveal the dynamics of the naturalization of roles and the intersectionality of their implications in the sociocultural context. On the one hand, the study shows how inequalities are reaffirmed for women or men, and, on the other hand, discloses the work done by the people involved, aiming at deconstructing those mechanisms and the preconceived ideas that surround them.

Keywords: Artisans; Gender stereotypes; Intersectionality; Women; Men.

\section{Estereótipos de gênero: Perspectivas em profissões de artesanato em Portugal}

Resumo: Este artigo explora os estereótipos de gênero em uma associação de artesãos em Lisboa, através da um estudo exploratório de caráter etnográfico, cujas contribuições explicam a origem e as transformações da profissão, bem como um conjunto de articulações que categorizam o feminino e o masculino. Os resultados expõem a dinâmica de naturalização dos papéis e a interseccionalidade de suas implicações no contexto sociocultural. O estudo mostra, por um lado, como as desigualdades são reafirmadas por ser uma mulher ou um homem e revela, por outro, o trabalho realizado pelas pessoas envolvidas, com o desejo de desconstruir esses mecanismos e as ideias preconcebidas que os cercam.

Palavras-chove: artesãos; estereótipos de gênero, interseccionalidade; mulheres; homens.

\section{Introducción}

El trabajo explora la perspectiva de artesanos de la Asociación de los Artesanos de la Región de Lisboa sobre los estereotipos de género, en un gremio conformado por 320 personas (de las cuales 18 participan en este estudio), dedicadas tradicionalmente a la creación de objetos artesanales en Portugal, agrupando diversos oficios y saberes en un contexto de herencia, transformación e innovación cultural. 
En ese ámbito, plantean, desde su perspectiva, los estereotipos de género que se reproducen, así como las transformaciones generacionales en torno a sus actividades. En primera instancia se recogen las percepciones en base a una entrevista' contextualizada en función del género, lugar y profesión, cuestionando el rol de lo femenino y masculino en ciertos procedimientos laborales y escenarios concretos.

A partir de esas experiencias, se identifican un conjunto de ideas, creencias y representaciones que dan significado a los mecanismos de reproducción, siendo así que estas opiniones estructuran la investigación en varias secciones, replanteando la visión del género en el campo artesanal. De ahí que variables como el origen, alteraciones, resistencias y hegemonía colonial del arte emerjan y se reflejen más adelante en cuatro categorías: Género, Familia, Trabajo y Desigualdad.

Los resultados propician una narrativa invertida de género, suscitando una reflexión a partir de los aportes de algunos autores, haciendo evidentes los mecanismos de poder con que opera "la dominación masculina" (Pierre BOURDIEU; Joaquín JORDÁ, 2000). Así, "Io masculino aparece en súperrepresentación frente a un sub-protagonismo de lo femenino" (Juan PLAZA; Carmen DELGADO, 2007, p. 8), o señalando que no hay "escritura femenina", en palabras de Monique Wittig (1980), citada en Judith Butler (2007, p.87), colocando a la mujer en un papel secundario y haciendo necesario desafiar esas formas ocultas que hacen un uso instrumental del lenguaje en la vida diaria como un dispositivo de poder, en el que"algunos mecanismos lingüísticos acentúan e invisibilizan a las mujeres y la valorización asimétrica en que es asociado lo masculino y lo femenino" (María PEREIRA, 2012, p.81).

\section{Metodología}

Este es un trabajo exploratorio de corte etnográfico, que analiza la perspectiva de género por medio de una entrevista semiestructurada realizada a 18 artesanos de Portugal ( 9 mujeres y 9 hombres). Algunas, y en mayor número, efectuadas en el marco de la Feria Internacional de Artesanos FIA 2017, un evento que se celebra en Lisboa anualmente, y otras en los sitios de trabajo de los artesanos.

La entrevista parte de un guion de preguntas que sirvió como referencia y punto de partida. Fue planteado a raíz de un encuentro con la coordinadora de AARLisboa ${ }^{2}$. En él se propone un repaso del contexto histórico actual y de trayectorias artesanales vinculadas a aspectos inherentes como la familia, economía y educación, en contraste con los intereses investigativos centrados en los estudios de género, proponiéndose bajo la siguiente estructura:

1. ¿Cómo aprendió el oficio que realiza actualmente? ¿Es una actividad a tiempo completo o parcial?

2. ¿Qué profesiones artesanales considera son más para las mujeres? ¿Y para los hombres? ¿Por qué?

¿Qué factores cree usted que influyen?

3. ¿Cuántas personas en su familia se han dedicado al trabajo artesanal? ¿Es una profesión que compatibiliza con otras actividades familiares o extra familiares?

4. ¿Cómo se distribuyen los recursos obtenidos de la profesión en la familia?

5. ¿La profesión que usted realiza ha sido ejercida tradicionalmente por mujeres o hombres?

6. ¿Con relación al pasado, considera que hubo alguna alteración en cuanto a las personas (sexo) que hacen este oficio?

7. ¿Ha presenciado algún conflicto entorno a su profesión (propio o ajeno) por el hecho de ser mujer u hombre? ¿Existe algún tipo de discriminación en su entorno de trabajo (género, origen u otro)?

8. ¿Le gustaría poder enseñar a hacer su oficio a otras personas?

9. ¿Cuáles cree que son las motivaciones para que otras personas decidan aprender o no la profesión?

Las entrevistas se realizaron en portugués, y una vez finalizado el trabajo de campo fueron traducidas al español por la investigadora, bajo el apoyo de miembros de la institución con competencias lingüísticas en portugués y castellano. No obstante, los aportes de las entrevistas son expuestos en castellano.

A partir de la metodología de análisis en la que se exploran los estereotipos de género, se pudo clasificar los resultados en base a cuatro categorías: Género, Familia, Trabajo y Desigualdad. A su vez, estas acogen subcategorías y componentes que operan y actúan interseccionalmente en las relaciones de vida de los artesanos, y que para el efecto son puntualizadas en los resultados (Tabla 1).

\footnotetext{
'Los textos de las entrevistas están escritos en castellano- lengua oficial de la investigadora, no obstante, las entrevistas se dieron en portugués- lengua oficial de los artesanos. La traducción fue realizada por la investigadora junto con miembros de la institución auspiciante, con el fin de ser publicado en castellano.

2 Siglas de la Asociación de Artesanos.
} 
Se incluyen también aportes que surgieron espontáneamente durante la investigación, afianzando la idea de que "perseguir preguntas en vez de respuestas permite encontrar significados" (Margarita DEL OLMO, 2003, p.193). Los datos recolectados fueron obtenidos voluntariamente, y se obtuvo el consentimiento de las/os artesanos para utilizar sus nombres, edad, profesión y origen, tanto para la transcripción de las entrevistas como para su publicación (Tabla

\begin{tabular}{l|l|l}
\hline \multirow{2}{*}{ Género } & Mujer & casa-sutileza - paciencia - niños - hogar \\
\cline { 2 - 3 } & Hombre & rua (calle) - fuerza-economía-mente \\
\cline { 2 - 3 } & Juventud & transición - tecnologia \\
\hline Familia & Tradición & influencia, herencia, aprendizaje \\
\hline Trabajo & Profesión & transformaciones - hobbie \\
\hline Desigualdad & Hegemonía & remuneración-artesanía-arte \\
\hline
\end{tabular}

2).

El acceso al campo fue propiciado por la colaboración del CIEG - ISCSP Universidad de Lisboa $^{3}$ para concertar las entrevistas. En principio los diálogos serían en visitas acordadas en los sitios de trabajo, sin embargo, estaba próxima la realización de la FIA 2017 y se propuso realizarlas durante este evento. Por tanto, la colaboración reúne un variado grupo de artesanos de diferentes regiones del país.

Tabla 2 - Codificación de Entrevistas - Artesanos de la Región de Lisboa

\begin{tabular}{|c|c|c|c|}
\hline Nombre & Edad & Oficio/ Profesión & Origen \\
\hline Ana & 45 & Bordadera & Lisboa \\
\hline Guilhermina & 53 & Miniaturista & Lisboa \\
\hline Madalena & 52 & Artista plástica, arte en piedras & Ferragudo \\
\hline Maria Ines & 26 & Artesana, costura para bebés & Sintra \\
\hline Florisbela & 62 & Cerámica figurativa & Matosinhos \\
\hline Maria Elisabete & 52 & Artesana en rendas de filé (encajes) & Felgueiras \\
\hline Martha & 36 & Tejedora en hilar & Amarante \\
\hline Alexandra & 44 & Bordados y serigrafía & Lisboa \\
\hline Isaura & 81 & Croché y telar & Lisboa \\
\hline Rui & 47 & Arte marinero & Salvaterre de Magos \\
\hline Paulo & 53 & Artes decorativas & Rio de Mouro \\
\hline Alexandre & 69 & Cesteria en papel periódico & Ribatejo \\
\hline Andrés & 48 & Ceramista & Lisboa \\
\hline Carlos & 52 & Cesteiro & Guarda \\
\hline David & 40 & Ceramista en azulejos & Lisboa \\
\hline Jose R & 65 & Zapatero & Almeirim \\
\hline Jose A & 61 & Artesano en piedras & Pampilhosa da Serra \\
\hline Nuno & 52 & Ceramista en barro & Queluz \\
\hline
\end{tabular}

Fuente: Propia. 
De esta forma, en los primeros acercamientos pude deshacerme de inseguridades propias, naturalizadas desde mi posición de extranjera, de mujer y con nociones básicas de la lengua portuguesa. Sin embargo, con el ingreso al mundo de los artesanos, ni el idioma, ni las diferencias impidieron el desarrollo armónico del trabajo, pues la raíz lingüística de ambas lenguas dio paso a un entendimiento fluido, pudiendo hacer uso de las dos lenguas simultáneamente.

Por otro lado, se trataba de un escenario complejo en el que se pusieron en juego todas las habilidades de las partes, en medio de un ambiente expuesto a imprevistos e interrupciones, pero con un alto ímpetu de colaboración. Así, el trabajo inicia con un diálogo amigable, explicando los objetivos e indicando que el ámbito de la investigación está vinculado a un trabajo más amplio de tesis doctoral, enfocado en la construcción de los imaginarios femeninos y masculinos en el sector artesanal de Manta-Ecuador.

Por consiguiente, los aportes que nacen de esta exploración contribuyen al enriquecimiento del marco de estudio, valorando la experiencia de Lisboa como un recurso que se transversaliza con la investigación en el sector artesanal en Ecuador.

\section{Género, perspectiva y teoría}

Esta investigación se sustenta en teorías que propician el diálogo y debate en torno a la desigualdad de género, dos de ellas recopiladas por Judith Lorber (2005) en su libro Gender Inequality, concretamente las corrientes postmodernas y el feminismo social. Estas teorías encuentran sustento en relación directa con los resultados de este trabajo, siendo acertado el uso de la teoría interseccional por lo multidisciplinario del entorno y las variables que se reflejan.

La idea del género construido social y culturalmente no deja de ser una cuestión latente en el sector artesanal, pues responde a patrones definidos por la sociedad y la tradición, siendo "necesario empezar a hablar de sexos, sexualidades y de géneros y no únicamente de género" (LORBER, 2005, p.5), así como también empezar a abordar la interseccionalidad del término en relación con otras áreas. La autora señala además que los roles de familia y trabajo, así como prácticamente otros aspectos sociales de la vida, son construidos en esas dos divisiones mayores, refiriéndose a las categorías clásicas (femenina y masculina), y dejando de lado otras formas de ser mujer u hombre. Además de las categorías binarias del sexo construidas socialmente, el argumento de esas corrientes posibilita otros ámbitos, experiencias y locaciones con relación al género, teniendo indiscutiblemente al alcance profesión y artesanado.

En el campo artesanal muy poco se ha hablado de género, aunque muchos estudios son adyacentes a la cultura en relación con los saberes ancestrales, profesiones y prácticas laborales (Rosabeth KANTER, 1977; Martha LAMAS, 2013; Ester HEREDIA et al., 2002; María POZZIO, 2012). No obstante, se acercan, pero no exploran el artesanado desde la mirada de género. En tal razón, lo recolectado es una aproximación de 18 voces artesanales, en contraste con corrientes que examinan el género desde otros campos y definiciones paralelas.

Los artesanos, partiendo de una visión propia, enuncian el discurso para lo femenino y masculino en base a códigos y normas que "no siempre están claramente explicitadas; a menudo se transmiten de manera implícita a través del lenguaje y otros símbolos" (LAMAS, 2013, p.3). Los estereotipos, aquí, están alimentados por la idea que ser mujer u hombre es nacer con un código de barras. "Se nace con la etiqueta puesta de niña o niño y dicha etiqueta se va a ir llenando de significados sociales y psicológicos que irán jalonando el curso de nuestras vidas" (María DURANGO, 2011 , p.55). Las diferencias encontradas en este contexto muestran cómo se conceptualiza y explica la definición de género, respondiendo a un sistema social que sesga roles en dos grupos (femenino y masculino) donde cohabitan "personas encarnadas como una marca de diferencia biológica, lingüística o cultural” (BUTLER, 2007, p.59), pero dejan fuera cuestiones específicas del ser humano.

Se plantea que "el género se entiende como un estatus social, una designación legal, y una identidad personal" (LORBER, 2005, p. 9) que puede cobijar tanto la multidisciplinariedad del ser como las propias individualidades en un sólo término y tal vez en un solo género, pues realmente "no hay dos géneros", y que "únicamente hay uno el femenino pues el 'masculino' no es un género. Porque lo masculino no es lo masculino sino lo general" (BUTLER, 2007, p. 28). Estas observaciones definen el género y hacen necesaria la incorporación de la interseccionalidad como herramienta para abordar las dualidades que lo gravitan y dividen en clases, etnias y desigualdades, permitiendo al individuo "flotar libremente, en un juego de significado y placeres"" (Raewyn CONNELL, 2002, p. 69).

El género en el área de las profesiones no es una cuestión aislada de la familia, ni de las estructuras económicas, es más bien otra demanda que posibilita valorar la diversidad y la heterogeneidad como partes fundamentales de convivencia y respeto, hallando otros puntos de encuentro, desencuentro y negociación.

"La interseccionalidad es una forma de comprender y analizar la complejidad en el mundo, en las personas y en las experiencias humanas" (Patricia COLLINS; Sirma BILGE, 2016, p. 9). Este

\footnotetext{
${ }^{3}$ Centro Interdisciplinar de Estudos de Gênero - ISCPS Universidad de Lisboa.
}

"Traducción propia al español de "Gender Short Construction". 
aporte refleja temas emergentes que abordan tópicos de clases social, sexualidad o educación, y que no son tratados directamente en el gremio.

[...] la división de género y sus normas de acompañamiento y las expectativas de roles se construyen en las principales instituciones de la sociedad, como la economía, la familia, el estado, la cultura, la religión y la ley, el orden social de género. (LORBER, 2005, p.9)

Estos procesos son parte de una interseccionalidad, que Collins y Bilge (2016) evocan como "una herramienta analítica usada, a menudo sin ser nombrada como tal". De otra mano, es notorio que la normalización de roles está definida y justificada en base a la tradición y a la no discusión.

Estas ideas estereotipadas se conciben a través de las generaciones, y resultan complejas para el debate. Se observan como hechos normalizados, no cuestionados, ni replanteados. Al no ser un tema de discusión, las diferencias pasan inobservadas y reproducen la cadena de desigualdad. Considerando así que "el género es tan endémico, porque a menos que veamos la diferencia, no podemos justificar la desigualdad"5 (Bárbara RISMAN, 2004, p. 430).

Si bien determinados hallazgos aún siguen ligados a un androcentrismo ambivalente en el que el hombre ha tenido un papel superior sobre la mujer, la relación dimensional de lo grande y pequeño, así como las desigualdades y diferencias entre el trabajo relacionado a la casa, a la calle y al poder.

"El paternalismo se estructura sobre la base del poder masculino" (Andrea VELANDIA; Javier ROZO, 2009, p. 22) como componente hostil se basa en la creencia que el hombre debería tener más poder que la mujer, y como componente benévolo se refiere al cuidado y el cariño que se les debe dar a las mujeres, por estar bajo la potestad masculina. Pero si bien es cierto, también se muestra una sustantividad maternalista, donde las mujeres han tenido mucho que ver, desde una subcategoría desarrollada dentro del hogar. De este modo, "unas mujeres-las madres-construyen ellas mismas la misoginia de los hombres, haciéndoles incapaces de establecer relaciones de igualdad con una mujer" (Camille LACOSTE-DUJARDIN, 1993, p. 147). Estos estereotipos responden a una línea patriarcal establecida en función al trabajo, propiciando que el hombre esté fuera de la casa para mantener y proteger la familia, explícitamente demostrando que el rango ha sido superior a lo femenino y a lo masculino, acentuando cada vez más los privilegios. En contraparte, la mujer se ha visto inducida al trabajo de casa (niños, alimentación, vestimenta) para que los hombres y aquellos que cuida sean atendidos. Reafirmando la idea de que "la fuerza masculina se descubre en el hecho de que prescinde de cualquier justificación: la visión androcéntrica se impone como neutra y no siente la necesidad de enunciarse en unos discursos capaces de legitimarla" (BOURDIEU; JORDÁ, 2000, p. 11).

Por otro lado, la influencia del código masculino determina, en algunos casos, las preferencias y los arquetipos en diversos contextos, la idealización de la figura paterna como el prototipo de hombre ideal, hace que estas ideas sean extrapoladas y reproducidas naturalmente. De este modo, la mujer se cobija en esos ideales; imitando un código y competiendo por alcanzar otros espacios, "la mujer se incorpora a la ciencia sólo con el código masculino y por lo tanto renunciando a su identidad femenina" (Eulalia SEDEÑO; Paloma ALCALÁ, 2001, p. 341).

\section{Resultados}

Artesanato es una profesión que envuelve un conjunto de prácticas naturalizadas, con características más o menos definidas para mujeres y hombres en un imaginario, que establece dualidades opuestas cuando se habla de género. Delimita rua ${ }^{6}$ para lo masculino y casa para lo femenino, en medio de relaciones asimétricas. En las respuestas obtenidas del guion y los diálogos, se aprecia que tanto la figura femenina como la masculina mantienen una estrecha relación con la tradición y con la idealización de los roles para ambas categorías, y que no sólo el hecho de ser socio culturalmente mujer u hombre los coloca en situación de ventaja o desventaja, sino que, a partir de esas dos variables, surgen otros enfoques.

El género, aquí, es visto como un aspecto que debe ser incorporado a las labores profesionales y familiares. Por un lado, se ha hecho evidente la necesidad de plantearlo como un tema prioritario, en el que todos participan y aspiran tener igualdad de oportunidades, y por otro, aparecen contradicciones en su discurso, en el que sus afirmaciones no coinciden con el reflejo de la realidad expresada a través de ellos.

Martha, tejedora de 36 años, dice que su "profesión no tiene género, no tiene diferencia, ha sido hecha también por mujeres de todas las edades". Su experiencia está fundamentada en casa, con su madre y otras mujeres de quiénes aprendió el oficio, el rol del hombre aquí era el de construir el telar (uso de maderas y fuerza).

5Traducción propia al español de "Gender as social structure".

'Palabra en portugués que significa calle. 
Andrés, ceramista de 48 años, menciona que "las profesiones de fuerza son más para los hombres, lo cual no quiere decir que las mujeres no lo hagan, pero para los hombres son más fáciles las profesiones que requieren de mucha fuerza es normal", enfatizando que no hay "distinciones para hombres ni para mujeres".

Aunque los colaboradores expresan que la profesión no tiene género, se construyen estereotipos indistintamente, para lo masculino por medio de la fuerza y para lo femenino la negación de trabajos que la requieran.

María Inés, artesana en costura de 26 años, menciona "hoy en día ya no hay profesión para hombre o para mujer. Los maridos ayudan en las ferias a montar, ya ayudan mucho, a pintar los muebles, ir con las grúas a los almacenes, buscar cosas y ayudarnos".

Las mujeres han reproducido lo que han hecho otras mujeres de su familia o su círculo de influencia, básicamente por tradición e imitación de los patrones generacionales. Isaura, tejedora en croché y telar, con 81 años, señala que "la tradición cuenta mucho, porque las mujeres veían los esbozos de los estilos de sus tías, o lo que sus antepasados hacían y ellas iban haciendo".

En el caso de los hombres, influye la tradición también, pero sus motivaciones están fundadas en fuerza y poder, y por la influencia de sus antepasados, sus abuelos trabajaban determinados objetos, por una cuestión de supervivencia, como lo señala Isaura.

Para Madalena, de 52 años, artista plástica, la influencia paternal fue decisiva, su padre es arquitecto. "La primera vez que comencé a hacer esto, fue por que vi a mi padre a quien le gustaba hacer pequeños trabajos y como nosotros éramos una familia media, yo comencé a imaginar y a utilizar materiales reciclados para hacer juguetes".

No obstante, esto varía en función de la época, de la familia, del rendimiento económico, del lugar y de las aspiraciones que tienen las demás personas para inclinarse o no por una profesión, independientemente del género.

Por ejemplo, José R, zapatero de 65 años, afirma: "Yo solo tengo un hijo y como sé que es una profesión en que no se gana mucho dinero, nunca he exigido a mi hijo seguirla". Se evidencia un amplio tejido social y la capacidad de análisis del género desde la influencia de clases sociales, familia y economía, influencias que son reflejadas en las cuatro categorías, subcategorías y componentes analizados en adelante.

\subsection{Género y espacios concretos. Calle, casa y profesión}

Una característica fundamental de la relación género-profesión es atribuida por las condiciones físicas de mujeres y hombres, afianzadas en un conjunto de opiniones que sesgan el género a la fuerza o debilidad como categoría hegemónica de poder.

Así, José, de oficio zapatero, señala que "para coser la suela del zapato, se requiere que la persona tenga más presencia física, más fuerza, por tanto, es un trabajo de los hombres". La calle y la casa se presentan aquí como escenarios en que mujeres y hombres se han desenvuelto en el campo profesional. La casa simboliza el lugar seguro para la mujer y el cual es otorgado por lo masculino como una forma de agradecimiento, este arquetipo modela a un hombre expuesto al trabajo forzado, pero además merecedor de los cuidados de la madre-mujer-artesana y ama de casa.

Rui, artesano marinero de 47 años, manifiesta: "los bordados eran más para mujeres y es un trabajo de casa y las otras profesiones para los hombres era un trabajo de calle. De casa y de rua".

Para que así pueda ser, algunas mujeres optan no sólo por quedarse en casa en medio de esa naturalización, sino que también buscan actividades que les permita cuidar del hogar, mantenerse ocupadas y ganar algo de dinero, aunque éste último no represente ingresos considerables. Ana, bordadora de 45 años, acota: "esta profesión suele ser más de mujeres, la mayor parte de bordadoras lo hace en casa, yo bordo en casa y al mismo tiempo cuido a los niños".

En la casa ocurren también otros tipos de actividades no visibilizadas que no cuentan como oficios remunerados. "Las mujeres sólo esperan realizarse a través del ejercicio de la maternidad en un marco patriarcal o bien a un servicio de un orden patrilineal que las madres contribuyen así a reproducir" (LACOSTE-DUJARDIN, 1993, p. 148).

Esta dinámica del género reafirma la fuerza asociada a lo masculino, al trabajo externo de casa, a la grandeza y a la relevancia social, minimizando el aporte de la fuerza laboral femenina. "Los ingresos obtenidos por un "varón proveedor" cubrían las necesidades de toda la familia, de manera que el trabajo femenino y juvenil eran complementarios, opcionales y no ameritaban el cubrimiento de las necesidades de subsistencia de estos grupos" (David DíEZ, 2007, p.7).

David, de 40 años, ceramista en azulejos, señala que "a lo mejor las mujeres tienen más paciencia para trabajos que necesitan más detalles, más paciencia". Observando cómo se expresa y justifica la delicadeza femenina, asegurando que, "los hombres hacen más los trabajos que sean para pensar, a lo mejor más complejos". Haciendo un uso instrumental de la mente, como característica propia de lo masculino. "A lo femenino suelen asociarse: emotividad, subjetividad, cuidado, sumisión, irracionalidad, intuición; y a lo masculino; racionalidad, 
objetividad, agresividad, creatividad, exteriorización" (SEDEÑO; ALCALÁ, 2001, p. 338).

José A, con 61 años y artesano en piedras, resalta esta afirmación, mencionando: "no conozco mujeres que hacen esto, porque la dureza del artefacto (refiriéndose a la piedra) sólo la trabajan hombres". Bajo el riesgo de caer en lo evidente, los entrevistados señalan que no hay diferencias y que las transformaciones han empezado a darse; sin embargo, las formas de trabajo e interrelación narradas sacan a flote ideas sesgadas, no sólo en contra de las mujeres y a favor de los hombres, sino que su naturalización oprime ambas categorías.

Es así, como David menciona que en la fábrica de azulejos; "las mujeres hacen cosas más pequeñas, más detalladas. Esa es la única diferencia que yo encuentro. Hace cuarenta años eran solo hombres, las mujeres sólo hacían las tacitas pequeñas - tazas para poner las olivas y después todo empezó a cambiar, hacían cosas pequeñas para el café también, para la leche".

Alexandre, 69 años de edad y cestero, también considera que "las mujeres se dedican más a cosas de diseños de red y los hombres más a una actividad más pesada. Pero ahora las actividades se conjugan tanto como para mujeres y como para hombres". Lo señalado, desde su opinión, "tiene que ver con la constitución física del hombre y de la mujer, las cosas más pesadas están asignadas más a hombres. Los textiles son más para mujeres, las maderas, los cueros es más para hombres".

Este criterio es compartido por Martha, tejedora de 36 años, "las maderas, las cosas más pesadas, el tipo de materias primas que son más duras entonces están más diseñadas para hombres. Las materias primas más delicadas están para mujeres".

Un resultado importante del estudio de género relativo a las profesiones, afianzado por los artesanos y respaldado en la idea de que "la base de género en las identidades laborales es notablemente duradera y no se modifica fácilmente por el incremento de mujeres u hombres en un determinado grupo ocupacional" (LAMAS, 2013, p. 5).

Estas ideas estereotipadas para asignar roles laborales se justifican en la tradición, que precisamente se mueve en las arenas de lo fuerte-débil y de la casa-calle. Sin embargo, algunas pistas demuestran que algunos oficios de fuerza eran y siguen siendo realizados en casa, por ejemplo, el tejido.

Para Isaura (tejedora), el oficio "primero era hecho por hombres. Es una profesión dura, de esfuerzo físico, más adelante, como era un trabajo hecho en casa, entonces eran las mujeres que se quedaban en casa a trabajar y los hombres buscaban empleos fuera".

El trabajo del hombre, como hemos visto, busca contextualizarse en la calle, a partir de esa determinación de espacios a los que se somete el género se crean las fronteras y los estereotipos para dos esferas que "no son suficientemente independientes una de otra, pero tampoco comparten metas idénticas o necesariamente usan los mismos medios para alcanzar el objetivos que comparten"7 (Margery WOLF, 1972, p. 169).

Finalmente, en esta categoría se introduce a la juventud, por la importancia que han dado los artesanos a esta etapa de vida. Hablan de ella, sin precisar el género en términos de una relación asexuada, como una etapa transitoria, y que "no merece una inversión significativa de preocupación y de recursos" (María LOZANO, 2003, p.13). Son considerados como un importante grupo de personas con habilidades deferentes y hasta cierto punto libre del legado cultural.

Los describen en base a parámetros marcados por el ritmo de vida actual, la tecnología y las aspiraciones laborales en función de lo económico, teniendo una mayor incidencia, en medio de un dominio provisto de facilidades y flexibilidades. "La juventud solamente adquiere su sentido en el futuro, y por ello a los jóvenes hay que contenerlos mientras llega su sensatez en la edad adulta" (LOZANO, 2003, p. 13).

"La juventud emerge como una capa social privilegiada" (DIEZ, 2007, p. 8). En este caso, no es cuestionada ni criticada en su decisión de participación en los oficios de sus padres o familia. Los artesanos plantean que los jóvenes buscan trabajo no con el objetivo y posibilidad de formarse para un futuro, sino por la necesidad de subsistir. Puesto que "el espejo de los adultos a su alrededor, está matizado por las relaciones de supervivencia y la búsqueda en ascenso económico" (DÍEZ, 2007, p. 4).

Carlos, artesano en cestas de 52 años, expresa que "la artesanía es una cosa que da mucho trabajo y poco dinero. Y la juventud no quiere mucho trabajo". Los artesanos valoran a este grupo, como un apoyo para el futuro, como "un agente importante de cambio social" (Juan REVILLA, 2001, p. 106), convirtiéndose en un soporte en el proceso de transición global, capaz de conocer y manejar nuevas tecnologías y redes de comercialización modernas.

Estas afirmaciones ubican a la juventud como la encargada de aportar las innovaciones, al integrarse en la estructura social. Ellos son protagonistas tanto de la reproducción como de la transformación social, y se convierten en un sujeto histórico con entidad propia, una especie de "minoría activa"8 (Serge MOSCOVICl, 1979).

Nuno, ceramista barro, de 52 años, anota que "los jóvenes empiezan a presentar ideas diferentes, lo cual es óptimo para nosotros, porque intercambiamos ideas y aprendemos algunas

${ }^{7}$ Traducción propia al español de - Uterine families and the women's community. 
cosas. Los jóvenes también nos enseñan algunas cosas".

[...] La juventud es un concepto cambiante que se reconstruye permanentemente; se reproduce en lo cotidiano [...] se construye en relaciones de poder definidas por las condiciones de alteridad, dominación, o desigualdad, unas veces superpuestas entre sí, otras veces definidas por el conflicto. (LOZANO, 2003, p. 17)

Una condición de dominación e influyente de manera significativa no sólo en las aspiraciones de los jóvenes en su etapa laboral, es la económica. En algunos casos resulta "un factor decisivo para un momento particular de la trayectoria, en el que se pondera el salario como recurso que les permite adquirir o lograr ciertos objetivos inmediatos" (Claudia JACINTO et al, 2005, p. 13).

Pues a decir por los artesanos, varios oficios y profesiones artesanales tienden a desaparecer, no sólo por la resistencia al cambio de métodos y tecnologías, sino también porque la mano de obra artesanal es mal pagada y los jóvenes buscan empleos mejor remunerados.

Carlos, subraya que: "no hay quien quiera aprender, esta es una cosa muy trabajosa, ahora nadie lo quiere hacer. Este oficio da mucho trabajo y poco dinero. Y la juventud no quiere mucho trabajo". Esta, es una de las preocupaciones con frecuencia manifestada, aunque no dejan de reconocer que sus esfuerzos y los del gobierno, intentan hacer que el oficio sobreviva.

\subsection{Género y familia}

La familia ha sido considerada tradicionalmente el núcleo de la sociedad desde un concepto más costumbrista. "En el plano social, propugna la cohesión intima de pocos miembros - familia nuclear- y la insolidaridad social" (Ana NAVARRO, 1982, p. 488). Se ha estructurado en base a un sistema patriarcal, donde el hombre-padre-hijo o hermano ejerce el poder y un rol protector en contra de aquello que viene de afuera, y donde la mujer-madre-hija-hermana, acepta la protección a cambio de trabajo capitalista no remunerado.

Si bien esos modelos persisten en determinados ámbitos, unos más resistente que otros, también es un prototipo de familia en crisis, "ha ido modificándose con las transformaciones influenciadas por los modelos económicos, culturales, educativos, políticos y sociales que se alejan cada vez de ese ideal instaurado" (Elizabeth JELIN, 2005, p. 394). Ello derivando en que ya no sólo hay un esquema único, ni en lo que respecta a los miembros, ni en los roles que desempeñan, existiendo "una multiplicidad de formas de familia y de convivencia" (JELIN, 2005, p. 395).

En estos artesanos, es notorio que la familia juega un papel relevante como base social, la familia ha estado presente en el aprendizaje de la profesión, en la memoria colectiva de sus sitios de origen y en las cuestiones que tercian sus actividades. Ana, en correspondencia, afirma; "este trabajo lo aprendí con mi madre, era un trabajo que pasó de generación en generación, siempre vi a mi madre hacerlo, entonces fue con ella que aprendí".

Martha, de su lado, asienta que no recuerda la edad en que aprendió el oficio "fue desde muy pequeñita", siguiendo también los modelos femeninos familiares. De otro lado, atribuyen que la influencia está determinada en los hábitos y comportamientos que se construyen dentro de la familia. Paulo, de 53 años, dedicado a las artes decorativas, considera que mucho "influyen las cuestiones familiares, un padre o una madre hace esto o lo otro, entonces el hijo lo hace".

Ana, como madre-trabajadora, piensa que "el factor que más influye es que nosotras podemos estar en casa con los niños, cuidarlos y al mismo tiempo bordar".

Desde el punto de vista de los hombres, se han obtenido afirmaciones planteadas también desde una influencia familiar, pero también de una implantación heredada, en la que el hombre se iniciaba muy niño al trabajo, por razones que iban desde reproducir el sistema patrilineal, colaborar en el hogar económicamente, o porque no tenía cabida en la casa (sitio de permanencia) tal como lo hacían las mujeres de la familia.

Para ellos, la familia también es ese nicho de transformación en sentido amplio. Reconocen la necesidad de equiparar las condiciones sociales de empleabilidad y accesos en los dos ámbitos (mujer / hombre). Han empezado a intercambiar roles a partir de la familia, las mujeres cada vez más están en las calles realizando trabajos que requieren fuerza física o mental, y los hombres en las casas llevando a cabo actividades laborales. En ambos casos hay una combinación ascendente de actividades compartidas, por el costo de la renta, muchos han optado por abrir negocios en sus hogares, incrementando la presencia masculina en esos espacios.

De otro lado, cuando se les ha preguntado sobre la compatibilidad del oficio con la familia, los hombres lo han considerado como un logro, y otros como una ayuda hacia las mujeres.

Paulo relata lo siguiente: "Yo ayudo en casa, yo ayudo a mi mujer y ella me ayuda a mí, hay un intercambio bueno para los dos, porque nos da tiempo".

Andrés afirma al respecto: "Tengo una hija, la llevo a la escuela y la voy a buscar, vamos a las actividades, nos acompañamos. Combino la profesión con la familia".

$\overline{{ }^{8} \text { Psicología de las Minorías Activas. }}$ 
Para David, no hay otra manera, con respecto a organizar los tiempos y compartir las tareas del hogar: "Es que tiene ser, porque tenemos dos hijos, con un año y otro de cinco, así que por más trabajo que tengamos, tenemos siempre que tener tiempo y compatibilizar con el trabajo de la casa".

Esta compatibilización de las tareas relacionadas entre profesión-familia también ha sido vista como un complemento, un medio de distracción en actividades culturales o como un nexo para permanecer más tiempo en familia.

Alexandre asienta que "compagina con otras actividades dedicadas al arte y la cultura". O José $\mathrm{R}$, quién considera necesario "hacer un espacio para tener un día fuera, ir a la playa con la familia".

José A, artesano en piedras, de 61 años de edad, señala que lo que obtiene de su trabajo, lo destina para viajes; "voy a viajes con mi esposa"; paralelamente, al igual que otras personas del gremio, tienen empleos como funcionarios públicos o privados. Se puede encontrar que determinadas funciones sociales son organizadas por los hombres (iniciativa, planificación), y en gran parte tiene que ver el aspecto económico, del cual la mujer (participante) dispone en menor proporción. Se afianza la interdependencia en torno al hombre proveedor económicamente, una vez que la mujer genera ingresos menores, como consecuencia de la desigualdad salarial.

Maria Elisabete cuenta que su marido "tiene un soporte financiero, pues yo sola no, mi marido es quien consigue conciliar nuestra vida financiera, vivir solo de esto no".

Las mujeres reconocen que los ingresos, en sus casos, no son suficientes para mantener el hogar, los recursos que obtienen del artesanato son utilizados para solventar gastos pequeños o para formación, como el caso de Alexandra: "Otros recursos son utilizados para formación, para decisión de procedimientos y materiales y el resto para casa". La brecha en términos salariales, además de mostrar mayores ingresos para lo masculino, plasma una distribución desigual en función del género, etnia y clase social.

\subsection{Género - Trabajo. Alteraciones y crisis}

El artesanato, definido por la Unesco ${ }^{9}$ como una expresión artística cuyos cimientos descansan en las tradiciones de una comunidad y descrito por los artesanos como un hobby, es una profesión que está muy lejos de ser un medio de sustento económico. Las aportaciones delinean un oficio motivado por una satisfacción personal-familiar, producto además de influencias y tradiciones.

Esto es confirmado por Paulo, quién menciona que es "funcionario público de profesión y mi hobby es el artesanato en 3D, artes plásticas". El hobby ha sido más de una vez expresado como complementariedad en sus entornos, se ha convertido en un vehículo movilizador de carácter colectivo conjugado con valores como la solidaridad, respeto, paciencia y empatía.

En esta categoría, se muestra que el trabajo está determinado desde dos márgenes, de hombres-artesanos-con empleo fijo y de mujeres-artesanas-amas de casa. Lo cual sigue propiciando espacios de desigualdad de género. El beneficio que supone a la mujer, en realidad, es una forma disfrazada de capitalismo, forjado en las bases patriarcales.

Cada vez hay más mujeres con empleos múltiples, además del trabajo que ya realizan en el hogar. "Si bien se trata de un oficio que implica saberes y habilidades transmitidos de generación en generación" (Paloma BONFIL; Blanca SUÁREZ, 2001, p. 588), también es cierto que ha estado subsistiendo a vulnerabilidades sociales, sujeto a desigualdades económicas, laborales y culturales que lo vinculan a un rico legado histórico, cuyo costo irónicamente continúa siendo mal pagado en los mercados.

David asienta que "una artesanía portuguesa es una artesanía genuina con calidad, única". Su trabajo pintando azulejos requiere técnica y precisión, él manifiesta que su calidad está garantizada, "nuestro trabajo no es para vender pilos a un euro, no es para eso, eso ha provocado muchos conflictos en Lisboa, en las ferias, esas artesanías cuando las llevan a casa y las usan se les va el color, las nuestras no".

José R, a la par, manifiesta su preocupación y apunta que "estamos en extinción, el trabajo real en mi área es lo que yo considero artesanato. iHacer zapatillas no!", "Aprender a hacer botas toma cuatro o cinco años, lleva bastante tiempo aprender esta profesión, tanto como hacer una licenciatura".

Hoy en día los mercados globalizados con productos industrializados hacen que el trabajo artesanal pierda valor, y que factores determinantes como la calidad, el tiempo y el significado del objeto queden de lado. Estas alteraciones son las que han provocado que cada vez menos personas se dediquen a la profesión, y que un gran número de personas que continúan en ella sean mujeres.

Martha anota que "antiguamente existían también algunos hombres, pero debido a que las actividades son mal pagadas, los hombres prefieren trabajar fuera".

${ }^{9}$ Artesanía y diseño. http://www.unesco.org/new/es/santiago/culture/creative-industries/crafts-design/ 
Desde otra perspectiva, en cambio hay quienes apuestan a la profesión y mantienen la motivación para continuar realizando el trabajo. Maria Elisabete, por ejemplo, ha comentado que "el tipo de encaje que yo hago es antiestrés, por lo tanto, es interesante y eso hace que en los workshops las personas se aproximen y tengan curiosidad de saber cómo se hace"

Para Guilhermina, "el artesanato es una cuestión financiera, que requiere divulgar lo que se sabe hacer, ahora estamos más abiertos también más ofertas de dinero a más condiciones financieras del país y nosotros tenemos más ofertas".

Florisbela, de 62 años, artesana en cerámica figurativa, es la única que señala se trata de "un oficio rentable, sino no estaría 43 años en esto, sino fuera rentable buscaría otro camino". Hace referencia además a una profunda pasión por la profesión, desde su experiencia personal junto a su esposo. Siendo así que "Ia diversidad de formas expresivas, incluyendo la artesanía, representa un espectro de la pluralidad humana y cada cual proviene de su propia trayectoria histórica" (Linda D' ÁMICO, 1991, p. 61).

Se ha notado un trabajo permanente por desmitificar los estigmas que enrolan a las mujeres y a los hombres en profesiones ligadas al género, pero también a la tradición, sus concepciones se alejan en gran medida de inventar tradiciones, ${ }^{10}$ pues "es esencialmente un proceso de formalización y ritualización, caracterizado por referencia al pasado, aunque sea por imposición de la repetición" (Eric HOBSBAWM, 1999, p. 4).

Alexandra lo certifica, diciendo "que cada vez hay más hombres aprendiendo a bordar, a hacer trabajos que antiguamente eran solo las mujeres que los hacían, como costura, el bordado, ese tipo de profesiones". Teniendo en cuenta, además, que mujeres y hombres están intercambiando roles laborales y experimentado esos cambios de manera favorable, Guilhermina concluye que "lo que si es cierto es que cada vez más las mujeres quieren aprender otros trabajos y los hombres se aventuran también por hacer otras cosas. No hay de más distinción".

Este grupo de personas ha empezado a reconocerse en otras áreas y no tiene problemas de adaptación, considera que ceñirse a la inmovilidad no sólo lo dejará sin profesión sino también sin la oportunidad de descubrirse en otras facetas, considerando entonces que "donde las viejas formas están vivas, las tradiciones no necesitan ser ni resucitadas ni inventadas" (HOBSBAWM, 1999, p. 7).

Isaura, en el mismo sentido, entrelaza esa tradición contando su experiencia: "Mi nieto es mi mentor y él es que me dice que vaya haciendo muñecos, porque él está más al pendiente de lo que a la mayoría gusta más a nivel de internet, juegos y esas cosas".

Otra de las interseccionalidades capaces de trabajar en oposición al androcentrismo del sistema, ya que "esas ideologías patriarcales que los han formado, oscurecen y distorsionan al sujeto y le dan muy pocos recursos para mirarse con nitidez" (Marcela LAGARDE, 1998, p. 37).

José A comenta que "el propio sistema de familia hacía que la mujer se quedara al cuidado de la casa y ayudara a la madre en cosas del hogar. Ahora eso ya no tiene cabida, la mujer tiene una profesión, cursos superiores y está más disponible a aprender cosas nuevas".

Florisbela piensa que "es la misma educación en la que había ese estigma que los hombres debían ir a trabajar a las fábricas y la mujer quedarse en casa y al mismo tiempo trabajando en el artesanato. Hoy en día ya no es igual". Tanto para ella como para muchos de los entrevistados, en la actualidad es más notorio el hecho de que el artesanato conjuga una dualidad de experiencias, sentimientos y conocimientos; "un artesano trabajando comunica emociones y participa en una dialéctica histórica" (D’ÁMICO, 1991, p. 67); siendo así que Madalena acota que adicionalmente a esas dualidades, "si tu trabajo brilla, no es importante si está un hombre o una mujer detrás de ese trabajo".

\subsection{Desigualdades}

La globalización ha provocado que este oficio, en el mundo capitalista, se vea reducido económicamente y haya generado el aumento de un mercado de imitaciones. El valor simbólico de consumir lo nuestro está sostenido por una racionalidad económica, evidenciándose que "buscar bienes y marcas extranjeras ha sido un recurso de prestigio y a veces una elección de calidad" (Néstor CANCLINI, 2012, p. 15). Estas circunstancias se mantienen vigentes, mientras que el trabajo artesanal pierde valor en el mercado, los esfuerzos de las minorías no decaen frente a una barrera de explotación y reduccionismo.

José R manifiesta que "en la actualidad la gente puede comparar un poco lo que es un zapato industrial y uno artesanal, fabricar o hacer unas botas para montar a caballo puede llevar entre once y trece horas. Para hacer unas botas normales 9 horas".

Se trata de un trabajo que involucra un conjunto de saberes, convirtiéndose en ese conocimiento propio, que urge ser insertado en el conocimiento universal. Concibiendo, por un lado, a "la cultura como un proceso de ensamblado multinacional, una articulación flexible de partes, un montaje de rasgos que cualquier ciudadano de cualquier país, religión o ideología

10Véase Inventando Tradiciones (HOBSBAWN, 1999) 
puede leer y usar" (CANCLINI, 2012, p. 16). Y "lo natural como la simple verdad de las cosas aprendidas naturalmente, hechos simples admitidos por hombres simples" (Clifford GEERTZ, 2001, p. 13).

José A alega que "esto es una cosa que nace con la persona, no se aprende, no se transmite. Es una cosa de espíritu, cualquier parte de la naturaleza tiene cosas que se pueden plasmar".

La desigualdad implícita de los sistemas culturales invisibiliza el saber popular que encierra el oficio, y por tanto las posibilidades de interconectar esos aprendizajes construidos en los márgenes de la academia y la ciencia. La utopía del interconocimiento es aprender otros saberes sin olvidar el de uno mismo. Esta es la idea de prudencia que subsiste bajo la "ecología de los saberes", formulada por (Boaventura DE SOUSA, 2010).

María Inés, con optimismo, señala que "ahora hay más chicas que empiezan a gustar de la costura, normalmente eran las personas más viejas que tenían ese hábito. Pero ahora cada vez más mujeres quieren aprender a coser, a tener una marca propia. Creo que las cosas ahora son mejores".

En un ámbito no lejano, aparece otro componente que deriva de esta categoría de análisis: el arte versus artesanato. La noción que diferencia arte y artesanato está basada en la idea colonizada de producción capitalista, considerando erróneamente que un trabajo hecho por personas que exponen en museos; difiere de los que se exponen en tiendas locales o en ferias, en valor y precio. Tanto el artesanato como el arte parecen haber adquirido algunas etiquetas que fomentan la desigualdad, vinculando cuestiones de raza, origen, sexo y clase social a su actividad, propiciando cualidades hegemónicas a unos y restándoselas a otros.

La forma con la que se asocia está ligada a la pobreza, a largas jornadas de trabajo manual, precios bajos, mientras que el arte es adscrito a cualidades de superioridad. "La acción de las élites intelectuales que construyen el estado moderno y lo elaboran en el museo a través de los objetos, narrando los orígenes y características básicas de la identidad nacional" (DEL OLMO, 2010 , p. 137), siendo aquellas que determinan los márgenes entre arte y artesanato. Esta crítica aclara el panorama nublado en el que se sumerge lo artesanal; apuntando a "los silencios, a lo que no se dice, rescatando otras voces, otras maneras de decir" (Marisa DE OLEAGA; Fernando MONGE, 2007, p. 276).

Durante este estudio, aparece la necesidad de reivindicar los derechos a sus creadores, de desmitificar la idea de que lo moderno tiene más valor que aquello que es creado por sus mentes y manos. Es un reclamo de los artesanos, quienes reconocen que entre el arte y el artesanato se ha construido una frontera, de un lado vislumbrando condiciones mínimas e informales de empleabilidad y disminución de precios, y del otro mejores condiciones y capacidad rápida de respuesta a precios altos, además de un reconocimiento artístico para aquellos que hacen arte. El artesanato, al igual que el motivo de los museos, se basa en la idea de "construir una sociedad multicultural basada en el conocimiento y respeto mutuo" (DEL OLMO, 2010, p. 138).

\section{Notas Finales}

Considerando que el género y los estereotipos que derivan de él no pueden ser trabajados desde ángulos distintos, se plantea encontrar otros medios que intersecten las experiencias nacidas desde las diferencias, en un principio mostradas como casos aislados desde la mirada social. Refiriéndonos a la familia, trabajo y género como categorías de estudio, estas relaciones han puesto en evidencia la cara estereotipada que afecta a dos grupos, a lo femenino, como es conocido desde los márgenes de la opresión, la asignación de roles en el hogar, la función maternal y a la ejecución de oficios y profesiones de carácter sutil, mientras que en el campo masculino se ha reafirmado la idea de la fuerza, el rol proveedor y el trabajo hecho fuera de casa.

Otro aspecto particular es la carga significativa de lenguaje estereotipado justificando el rol de la mujer, al mismo tiempo que se la coloca en escenarios masculinos se continúan reproduciendo privilegios patriarcales que acrecientan las distancias. Esta incursión en nuevas facetas no es suficiente para hablar de igualdad desde lo femenino.

El género no es una cuestión de dos bordes, sino de seres humanos interconectados bajo los estándares que promueven la diversidad y el acceso a igualdad de oportunidades, valorando y haciendo uso de la homogeneidad y de las diferencias. En ese sentido, resulta oportuno mencionar que "el género no siempre se constituye de forma coherente o consistente en contextos históricos distintos, porque se entrevera con modalidades raciales, de clase, etnias, sexuales y regionales de identidades discursivamente constituida" (BUTLER, 2007, p. 49).

Ante esta inserción, es conveniente establecer modelos de formación formal (educación) e informal (cotidianeidad) con un enfoque interseccional que, además de la propuesta de (BUTLER, 2007), vincule el género como transversalidad, perspectiva y punto de encuentro. Un modelo que plantee escenarios en constante movimiento, ajustables a las identidades que nazcan de los géneros, pero que además respondan a las variaciones culturales del entorno, conflictos, realidades 
y alteraciones. Estos nuevos sitios de convivencia podrían ayudar a desfragmentar ideas estereotipadas y prejuicios que se reproducen de manera naturalizada, puesto que ya no sólo se trata de un espacio que da voz a los artesanos, sino también a un grupo de actores que inciden en la práctica: academia, sociedad civil y estado, garantizando esfuerzos unificados en dirección a un mismo camino.

Finalmente es necesario cuestionar las razones que legitiman desigualdades sociales en el momento actual, y empezar a trabajar en acciones transfiguradas que desvirtúen actos, vocabulario, paradigmas, prejuicios y prácticas sexistas. Para el fin, se requerirá una herramienta poderosa, capaz de lograr esas transformaciones sociales, por medio de la generación de "una conciencia crítica, de una actitud de cuestionamiento, de una práctica de la concienciación". (Patricia MATA; Belén BALLESTEROS; María PADILLA, 2012, p. 133) reflejada en un sistema educativo inclusivo, con miras a una ciudadanía para la igualdad de género.

\section{Referencias}

BONFIL, Paloma; SUÁREZ, Blanca. De la tradición al mercado: microempresas de mujeres artesanas. México: Pemsa, 2001.

BOURDIEU, Pierre; JORDÁ, Joaquín. La dominación masculina, v. 3. Barcelona: Anagrama, 2000.

BUTLER, Judith. El género en disputa: el feminismo y la subversión de la identidad. USA: Paidós, 2007.

CANCLINI, Néstor. Consumidores y ciudadanos. Grijalbo, 2012.

COLLINS, Patricia; BILGE, Sirma. Intersectionality. John Wiley \& Sons, 2016.

CONNELL, Raewyn. Gender Short Introductions. Cambridge: Polity Press, Blackwell Publishers, 2002.

D’ÁMICO, Linda. "Artesanía e identidad cultural: Una cuestión de historia, ideología y elección". Sarance, Otavalo, v. 15, p. 61-70, 1991.

DE OLEAGA, Marisa; MONGE, Fernando. "El museo de América: modelo para armar". Historia y Política, p. 276, 2007.

DEL OLMO, Margarita. (Coord.). Dilemas Éticos En Antropología: Las Entretelas Del Trabajo de Campo Etnográfico. España: Trota, 2010. p. 137. ISBN 978-84-9879-171-6

DEL OLMO, Margarita. "La construcción de la confianza en el trabajo de campo. Los límites de la entrevista dirigida". Revista de dialectología y tradiciones populares, La Rioja, v. 58, n. 1, p. 193, 2003.

DíEZ, David. "Juventud, género y trabajo: una mirada a formas de empleo juvenil en Colombia". Revista Controversia, Medellín, n. 188, p. 57-85, 2007.

DURANGO, María. "Estudio de las erogeneidades y defensas desarrolladas por un grupo de mujeres políticas en el Ecuador". Subjetividad y procesos cognitivos, Ciudad Autónoma de Buenos Aires, v. 14 , n. 1, p. 55-78, 2010. ISSN 1852-7310

GEERTZ, Clifford. Conocimiento local. Buenos Aires: Paidós, 2001.

HEREDIA, Ester; SARRIÓ, Maite; RAMOS, Amparo; CANDELA, Carlos. "Más allá del techo de cristal. Diversidad de género". Sostre de Vidre, Valencia, n. 40, p. 55-68. 2002. ISSN $1137-5868$

HOBSBAWM, Eric. "Inventando tradiciones". MÉNDEZ GALLO, Pablo (Trad.). Revista BitARTE, San Sebastián, n. 18, p. 39-53, 1999.

JACINTO, Claudia, et al. "Jóvenes, precariedades y sentidos del trabajo". In: Ponencia presentada en el 7 Congreso Nacional de Estudios del Trabajo. Buenos Aires, Argentina, 2005.

JELIN, Elizabeth. "Familia y género: notas para el debate". Revista Estudos Feministas, Florianópolis, v. 3, n. 2, p. 394-413, 1995.

KANTER, Rosabeth. "Some effects of proportions on group life: Skewed sex ratios and responses to token women." American Journal of Sociology, Chicago, v. 82, n. 5, p. 965-990, 1977.

LACOSTE-DUJARDIN, Camille. Las madres contra las mujeres: patriarcado y maternidad en el mundo árabe. España: Universitat de València, 1993. 
LAGARDE, Marcela. Identidad genérica y feminismo. Sevilla: Instituto Andaluz de la Mujer, 1998.

LAMAS, Martha. El género. La construcción cultural de la diferencia sexual. Costa Rica: Grupo Editorial Miguel Ángel Porrúa, 2013.

LORBER, Judith. Gender inequality: Feminist theories and politics. USA: Oxford University Press, 2005.

LOZANO, María. "Nociones de Juventud". Última década, Viña del Mar, n. 18, p. 11-19, 2003.

MATA, Patricia; BALLESTEROS, Belén; PADILLA, María. "Ciudadanía crítica, participativa y transformadora: experiencias de aprendizaje." In: Simposio Internacional de Didáctica de las Ciencias Sociales (2012), 131-138. España. Asociación Universitaria de Profesorado de Didáctica de las Ciencias Sociales: Díada, 2012. p. 133.

MOSCOVICl, Serge. Psicología de las minorías activas. Madrid: Morata, 1981.

NAVARRO, Ana María. "El feminismo y la familia". In: Cuestiones fundamentales sobre matrimonio y familia: II Simposio Internacional de Teología Universidad de Navarra, EUNSA, 1982, p. 481-492.

PEREIRA, María. Fazendo género no recreio: a negociação do género em espaço escolar. Lisboa: Imprensa de Ciências Sociais, 2012.

PLAZA, Juan; DELGADO Carmen. Género y Comunicación. Madrid: Editorial Fundamentos, 2007. ISBN 978-84-245-1 136-4

POZZIO, María. "Análisis de género y estudios sobre profesiones: propuestas y desafíos de un diálogo posible-y alentador." Sudamérica: Revista de Ciencias Sociales, Mar del Plata, n. 1, p. 99-129, 2012.

REVILLA, Juan. "La construcción discursiva de la juventud: lo general y lo particular". Papers: revista de sociologia, Madrid, v. 63/64, p. 103-122, 2001.

RISMAN, Bárbara. "Gender as a social structure: Theory wrestling with activism". Gender \& society, v. 18, n. 4, p. 429-450, 2004.

SANTOS, Boaventura de Sousa. Descolonizar el saber, reinventar el poder. Montevideo: Ediciones Trilce, 2010.

SEDEÑO, Eulalia; ALCALÁ, Paloma. (Coords.). Ciencia y género. Colección Philosophica Complutense, v. 15. Madrid: Editorial Complutense, 2001.

UNESCO. "Artesanía y diseño". Chile. Disponible en http://www.unesco.org/new/es/santiago/culture/ creative-industries/crafts-design/. Acceso el 09/09/2017.

VELANDIA, Andrea; ROZO, Javier. "Estereotipos de género, sexismo y su relación con la psicología del consumidor". Psychologia - Avances de la disciplina, Bogotá, p. 17-34, 2009.

WITTIG, Monique. "The straight mind”. Feminist Issues, v. 1, n. 1, p.103-11, 1980.

WOLF, Margery. “Uterine Families and the Women's Community”. In: WOLF, Margery. Women and the Family in Rural Taiwan. Stanford University Press, 1972, p. 166-169.

Fanny Tubay Zambrano (fannytubay@hotmail.com) es doctoranda del Programa de Educación en la línea de Estudios Interculturales - CIEG Centro Interdisciplinar de Estudios de Género - ISCSP Universidad de Lisboa, Portugal. Docente investigadora en temas de educación, interculturalidad, género y saberes ancestrales, con estudios de maestría en Educación Intercultural, actualmente es estudiante del Programa de Doctorado en Educación, en la línea de estudios interculturales. Estancia doctoral en Portugal, en el CIEG del ISCSP, en la que participó en investigaciones sobre género en el ámbito de las profesiones, específicamente en artesanato. También investiga el género como estructura social, la construcción de la femineidad y la masculinidad en el sector artesanal en Ecuador. 


\section{COMO CITAR ESTE ARTÍCULO, DE ACUERDO CON LAS NORMAS DE LA REVISTA:}

TUBAY, Fanny Monserrate Tubay. "Estereotipos de género: Perspectivas en profesiones artesanales de Portugal”. Revista Estudos Feministas, Florianópolis, v. 27, n. 2, e54182, 2019.

\section{CONTRIBUCIÓN DE AUTORÍA}

No se aplica

\section{FINANCIACIÓN}

No se aplica

\section{CONSENTIMIENTO DE USO DE IMAGEM}

No se aplica

APROBACIÓN DE COMITÉ DE ÉTICA EN INVESTIGACIÓN

No se aplica

\section{CONFLICTO DE INTERESSES}

No existe conflito de intereses

\section{LICENCIA DE USO}

Este artigo está licenciado sob a Licença Creative Commons CC-BY Internacional. Com essa licença você pode compartilhar, adaptar, criar para qualquer fim, desde que atribua a autoria da obra.

\section{HISTÓRICO}

Recebido em 22/1 1/2017

Reapresentado em 13/09/2018

Aprovado em 19/10/2018 